\title{
Hadronic Spectroscopy from the Lattice: Glueballs and Hybrid Mesons
}

\author{
Chris Michael ${ }^{\mathrm{a}}$ \\ a Division of Theoretical Physics, Department of Mathematical Sciences, \\ University of Liverpool, Liverpool L69 3BX, UK
}

Lattice QCD determinations appropriate to hadron spectroscopy are reviewed with emphasis on the glueball and hybrid meson states in the quenched approximation. Hybrids are discussed for heavy and for light quarks. The effects of sea quarks (unquenching) are explored.

\section{INTRODUCTION}

Quantum Chromodynamics is generally acknowledged to be the theory of hadronic interactions. Its perturbative features are well understood and provided the main motivation for its adoption. To give a complete description of hadronic physics, it is essential to develop nonperturbative methods to handle QCD. QCD is a quantum field theory which needs to be regulated in order to have a well defined mathematical approach. Inevitably such a regularisation will destroy some of the symmetries of QCD. For example dimensional regularisation is often used in perturbative studies and it breaks the 4dimensional Lorentz invariance. Similarly lattice regularisation as proposed by Wilson [1] breaks Lorentz invariance since a hypercubic lattice of space-time points is invoked. The key feature of Wilson's proposal is that gauge invariance is exactly retained. Then the approach to the continuum limit (as the lattice spacing $a$ is reduced to zero) can be shown to be well defined. Using Monte Carlo methods to explore lattice QCD, reliable predictions can now be made for continuum quantities as I shall discuss. Moreover, in the continuum limit, the Lorentz invariance is found to be fully restored.

Lattice QCD needs as input the quark masses and an overall scale (conventionally given by $\left.\Lambda_{Q C D}\right)$. Then any Green function can be evaluated by taking an average of suitable combinations of the lattice fields in the vacuum samples. This allows masses to be studied easily and matrix elements (particularly those of weak or elec- tromagnetic currents) can be extracted straightforwardly. Unlike experiment, lattice QCD can vary the quark masses and can also explore different boundary conditions and sources. This allows a wide range of studies which can be used to diagnose the health of phenomenological models as well as casting light on experimental data.

It is worth recalling the weakness as well as the strength of the lattice approach to QCD. Any quantity which can be expressed as a vacuum expectation value of fields can be extracted straightforwardly in lattice studies. Thus masses and matrix elements of operators can be determined. What is not so easy is to explore hadronic decays. This is difficult because the lattice, using Euclidean time, has no concept of in and out states. About the only feasible strategies are to evaluate the mixing between states of the same energy - so giving some information on on-shell hadronic decay amplitudes, or to make a model dependent analysis [2] of lattice results.

One very special case is of considerable interest: this is quenched QCD where the sea-quark masses are taken as infinite. This suppresses quark loops in the vacuum completely, leaving just the full non-perturbative gluonic interactions. This gluonic vacuum turns out to reproduce most of the salient features of QCD. It is also a very convenient approximation to use for comparison with phenomenological models. Quenched QCD is computationally rather easy to study and the precise results allow the continuum limit to be extracted reliably. Studies with sea quark effects included (known as dynamical fermion studies) are computationally much more demanding. I 
discuss the current situation in this area in the last section.

\section{GLUEBALLS}

Glueballs are defined to be hadronic states made primarily from gluons. The full nonperturbative gluonic interaction is included in quenched QCD. In the quenched approximation, there is no mixing between such glueballs and quark - antiquark mesons. A study of the glueball spectrum in quenched QCD is thus of great value. This will allow experimental searches to be guided as well as providing calibration for models of glueballs.

In principle, lattice QCD can study the meson spectrum as the sea quark mass is decreased towards experimental values. This will allow the unambiguous glueball states in the quenched approximation to be tracked as the sea quark effects are increased. It may indeed turn out that no meson in the physical spectrum is primarily a glueball - all states are mixtures of glue, $q \bar{q}$, $q \bar{q} q \bar{q}$, etc. Studies conducted so far show no significant change of the glueball spectrum as dynamical quark effects are added - but the sea quark masses used are still rather large [3].

In lattice studies, dimensionless ratios of quantities are obtained. To explore the glueball masses, it is appropriate to combine them with another very accurately measured quantity to have a dimensionless observable. Since the potential between static quarks is very accurately measured from the lattice (see the next section for more details), it is now conventional to use $r_{0}$ for this comparison. Here $r_{0}$ is implicitly defined by $r^{2} d V(r) / d r=1.65$ at $r=r_{0}$ where $V(r)$ is the potential energy between static quarks which is easy to determine accurately on the lattice. In practice $r_{0}$ may be related to the string tension $\sigma$ by $r_{0} \sqrt{\sigma}=1.18$. Taking the conventional value of $\sqrt{\sigma}=0.44 \mathrm{GeV}$ then yields $r_{0}=2.68 \mathrm{GeV}^{-1}=0.53 \mathrm{fm}$. Taking the potential $V(r)$ from $b \bar{b}$ spectra suggests that $r_{0} \approx 0.5 \mathrm{fm}$ which is a very similar estimate.

Theoretical analysis indicates that for the quenched approximation the dimensionless ratio $m r_{0}$ will differ from the continuum limit value by

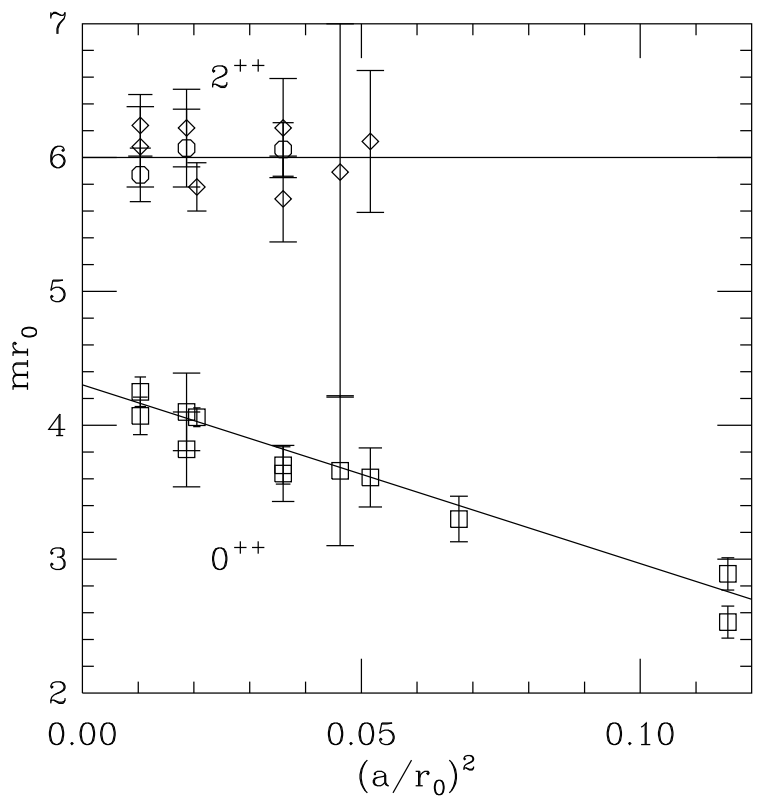

Figure 1. The value of mass of the $J^{P C}=0^{++}$ and $2^{++}$glueball states from refs [四 [7] in units of $r_{0}$ where $r_{0} \approx 0.5 \mathrm{fm}$. The $T_{2}$ and $E$ representations are shown by octagons and diamonds respectively and their agreement indicates the restoration of rotational invariance for the $2^{++}$ state. The straight lines show fits describing the approach to the continuum limit as $a \rightarrow 0$.

corrections of order $a^{2}$. Thus in Fig. 1 the masses are plotted versus the lattice spacing $a^{2}$ for the $J^{P C}=0^{++}$and $2^{++}$glueballs. The straight lines then show the continuum limit obtained by extrapolating to $a=0$. As can be seen, there is essentially no need for data at even smaller $a$ values to further fix the continuum value. The values shown correspond to $m\left(0^{++}\right) r_{0}=4.33(5)$ and $m\left(2^{++}\right) r_{0}=6.0(6)$. Since several lattice groups [4] have measured these quantities, it is reassuring to see that the purely lattice observables are in excellent agreement. The publicised difference of quoted $m\left(0^{++}\right)$from UKQCD [6] and GF11 [7] comes entirely from relating quenched lattice measurements to values in $\mathrm{GeV}$ as I now discuss. 
In the quenched approximation, different hadronic observables differ from experiment by factors of up to $10 \%$. Thus using one quantity or another to set the scale, gives an overall systematic error. Here I choose to set the scale by taking the conventional value of the string tension, $\sqrt{\sigma}=0.44 \mathrm{GeV}$, which then corresponds to $r_{0}^{-1}=373 \mathrm{MeV}$. An overall systematic error of $10 \%$ is then to be included to any extracted mass. This yields $m\left(0^{++}\right)=1611(30)(160) \mathrm{MeV}$ and $m\left(2^{++}\right)=2232(220)(220) \mathrm{MeV}$ where the second error is the systematic scale error. Note that these are glueball masses in the quenched approximation - in the real world significant mixing with $q \bar{q}$ states could modify these values substantially.

Recently a lattice approach using a large spatial lattice spacing with an improved action and a small time spacing has been used to study glueball masses. The results [8] are that $m\left(0^{++}\right) r_{0}=$ $3.98(15), m\left(2^{++}\right) r_{0}=5.85(2), m\left(1^{+-}\right) r_{0}=$ $7.21(2)$ and $m^{\prime}\left(2^{++}\right) r_{0}=8.11(4)$. There remains a small discrepancy with the result for the $0^{++}$ glueball obtained above $\left(m\left(0^{++}\right) r_{0}=4.33(5)\right)$ from lattice spacings much closer to the continuum limit. This has recently been explored in more detail [9] and modifications proposed to the technique which allow better control of the error in extrapolating to the continuum limit.

I have focussed on the scalar and tensor glueball results because these are the lightest and best measured states in lattice studies. The glueball spectrum has been extracted for all $J^{P C}$ values 5 .6. Some of these results are shown in Fig. 2. One signal of great interest would be a glueball with $J^{P C}$ not allowed for $q \bar{q}-$ a spinexotic glueball or oddball. These states are shown in Fig. 2 to be high lying: at least above $2 m\left(0^{++}\right)$. Thus they are likely to be in a region very difficult to access unambiguously by experiment.

The only other candidate for a relatively light glueball is the pseudoscalar. Values quoted of $m\left(0^{-+}\right) r_{0}=5.6(6), 7.1(1.1)$ and 5.3(6) from refs [5.60 suggest an average of $6.0(1.0)$, close to the tensor glueball mass. This is confirmed by a study using asymmetric lattices [9] which obtains a pseudoscalar glueball mass ratio to the tensor glueball of $m\left(0^{-+}\right) / m\left(2^{++}\right)=1.17(9)$.
Within the quenched approximation, the glueball states are unmixed with $q \bar{q}, q \bar{q} q \bar{q}$, etc. Furthermore, the $q \bar{q}$ states are 'magically mixed' in the quenched approximation. Once quark loops are allowed in the vacuum, for the favour-singlet states of any given $J^{P C}$, there will be mixing between the $s \bar{s}$ state, the $u \bar{u}+d \bar{d}$ state and the glueball. For the $0^{++}$case, as a guide to this mixing, the quenched mass spectrum of $q \bar{q}$ states has been determined on a lattice and the scalar mesons are found to lie somewhat lighter than the tensor states 10 . These $2^{++}$mesons are experimentally approximately 'magically mixed' so will be quite close to the quenched mass determination. This suggests that the quenched scalar masses from the lattice are at around $1.2 \mathrm{GeV}$ and $1.5 \mathrm{GeV}$. An independent study [11.12] suggests that the scalar $s \bar{s}$ state is about $200 \mathrm{MeV}$ lighter than the glueball which is a broadly compatible conclusion. Thus the glueball, at around 1.6 GeV, lies heavier than the $q \bar{q}$ scalar states.

A first attempt 12] has been made to measure, on a quenched lattice, the mixing matrix element between the glueball and the scalar $q \bar{q}$ states. The mixing matrix element between the $s \bar{s}$ meson and the glueball is extracted as $E(s)=43(31) \mathrm{MeV}$. The mixing matrix elements with the glueball are found to be similar for light and $s \bar{s}$ quarks (actually $E(u, d) / E(s) \approx 1.2)$. With this lattice input, a mixing model 13, 12] allows the content of the experimental scalar states at 1390, 1500 and 1710 $\mathrm{MeV}$ to be assigned. Other, more phenomenological mixing models have also been proposed [14]. In these mixing scenarios none of the experimental states is predominantly an unmixed glueball.

As well as this mixing of the glueball with $q \bar{q}$ states, there will be mixing with $q \bar{q} q \bar{q}$ states which will be responsible for the hadronic decays. Because of the limitations of lattice techniques, it is only possible to estimate these decay matrix elements when the initial and final states have the same energy and this has been applied to the decay matrix element between a $0^{++}$glueball and two pseudoscalar $q \bar{q}$ mesons. This then allows an estimate of the width which would arise when going beyond the quenched approximation. A first attempt to study this 15] yields estimated widths of order $100 \mathrm{MeV}$. Even though this lattice cal- 


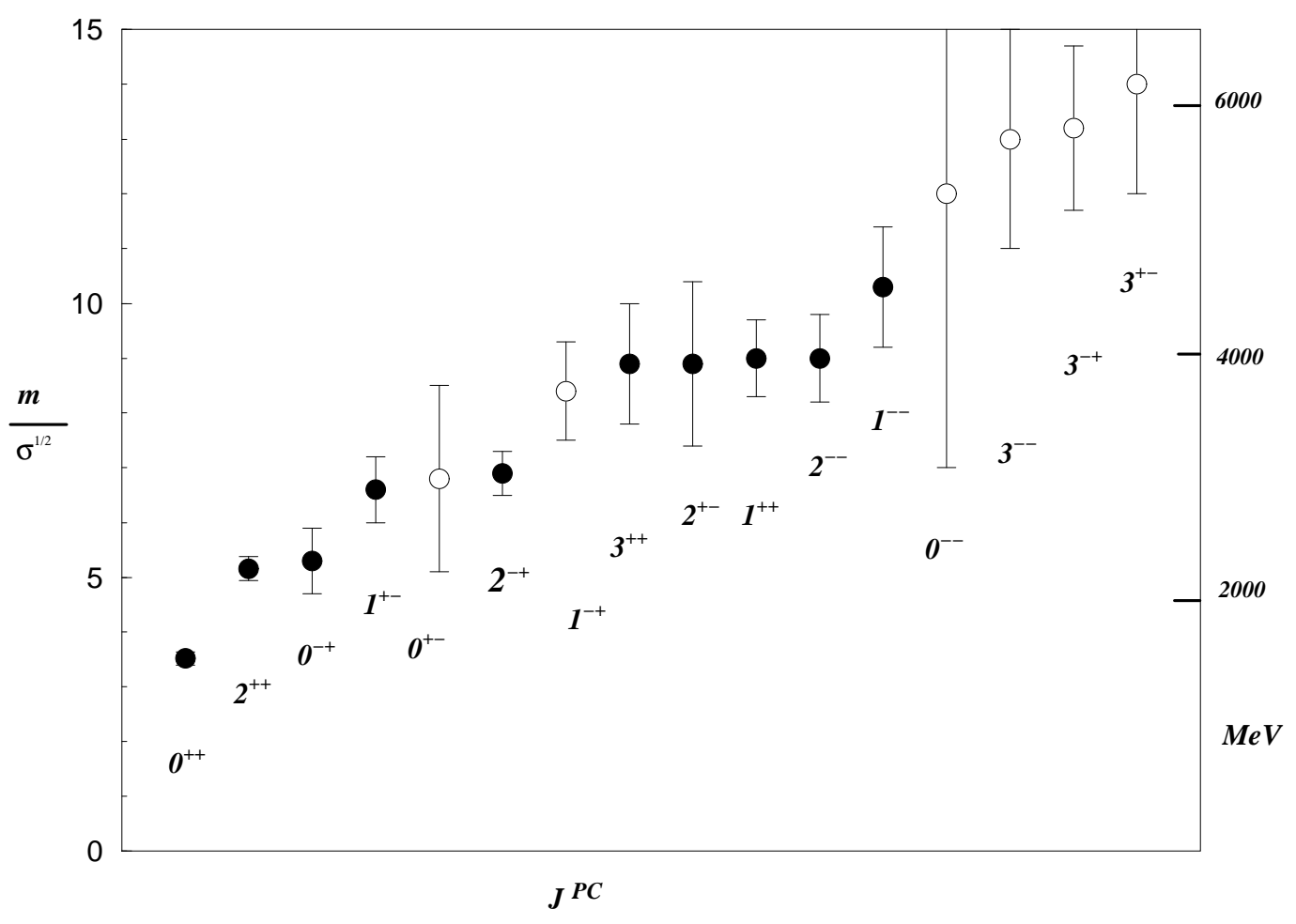

Figure 2. The mass of the glueball states with quantum numbers $J^{P C}$ from ref[6]. The scale is set by $\sqrt{\sigma} \approx 440 \mathrm{MeV}$ which yields the right hand scale in $\mathrm{MeV}$. The solid circles represent mass determinations whereas the open circles are upper limits. Note that excited states in several cases have been determined, such as $m^{\prime}\left(0^{++}\right) / \sqrt{\sigma}=6.3(4)$ from ref[5].

culation is very exploratory, it does indicate that very wide widths to two pseudoscalars are not expected. A more realistic study would involve taking account of mixing with the $n \bar{n}$ and $s \bar{s}$ scalar mesons as well as the decay channels.

\section{HEAVY QUARK INTERACTIONS}

In the limit $m_{Q} \rightarrow \infty$, the heavy quark effective theory describes a universal behaviour. For finite $m_{Q}$, corrections of order $1 / m_{Q}$ are expected. The simplest way to study the heavy quark limit on a lattice is to use static quarks. The potential energy $V(R)$ between a static quark and antiquark at separation $R$ is readily obtained. Then for heavy quarks, one may solve for the spectra in this potential using the Schrödinger equation in the adiabatic approximation. This is expected to be a good approximation for determining the spectrum of $b \bar{b}$ mesons. The quenched lattice potential is well measured and is found to have a form parametrised by

$V(R)=V_{0}-\frac{e}{R}+\sigma R$

where $e$ is the coefficient of the Coulomb term and $\sigma$ is the string tension. This expression shows that the potential continues to increase as $R$ is increased - this is confinement.

A comparison from ref 16 of the spectrum in the quenched lattice potential with the $\Upsilon$ states is shown in Fig. 3. The lattice result is quali- 


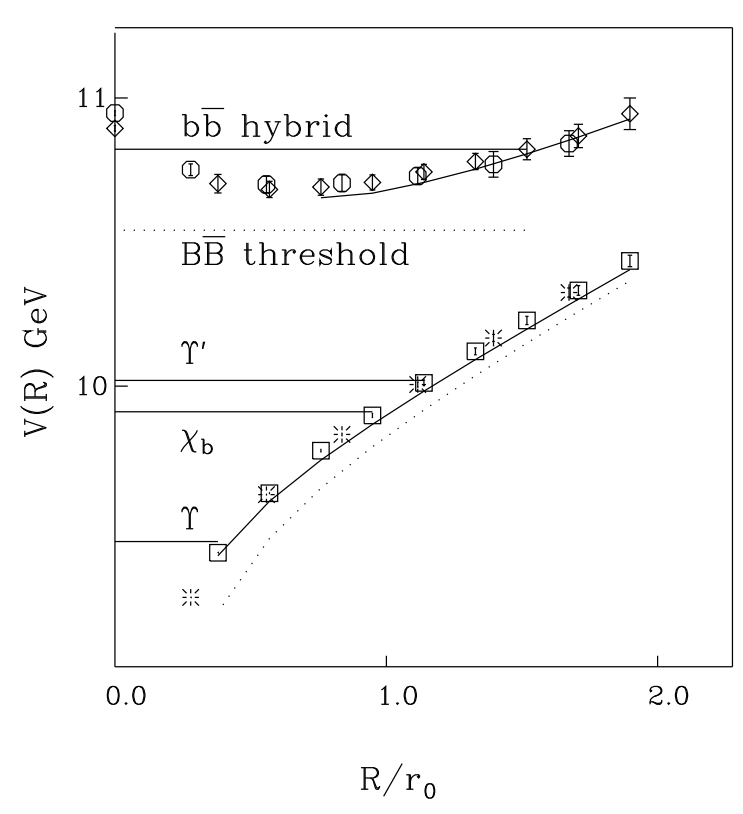

Figure 3. Potentials $V(R)$ in $\mathrm{GeV}$ between static quarks at separation $R$ (in units of $r_{0} \approx 0.5 \mathrm{fm}$ ) for the ground state (square and $*$ ) and for the $E_{u}$ symmetry which corresponds to the first excited state of the gluonic flux (octagons and diamonds). Results from the quenched calculations of ref[16] are shown by symbols corresponding to different lattice spacings. For the ground state potential the continuous curve is an interpolation of the lattice data while the dotted curve with enhanced Coulomb term fits the spectrum and yields the masses shown. The lightest hybrid level in the excited gluonic potential is also shown.

tatively similar to the experimental $\Upsilon$ spectrum. The main difference is that the Coulombic part $(e)$ is effectively too small (0.28 rather than 0.48$)$. This produces [16] a ratio of mass differences $(1 P-1 S) /(2 S-1 S)$ of 0.71 to be compared with the experimental ratio of 0.78 . This difference in $e$ is understandable as a consequence of the one gluon exchange (Coulombic) force at short distances which would be increased by $33 /\left(33-2 N_{f}\right)$ in perturbation theory in full QCD compared to quenched QCD. We will return to discuss this.
The situation of a static quark and antiquark is a very clear case in which to discuss hybrid mesons which have excited gluonic contributions. A discussion of the colour representation of the quark and antiquark is not useful since they are at different space positions and the combined colour is not gauge invariant. A better criterion is to focus on the spatial symmetry of the gluonic flux. As well as the symmetric ground state of the colour flux between two static quarks, there will be excited states with different symmetries. These were studied on a lattice [17] and the conclusion was that the $E_{u}$ symmetry (corresponding to flux states from an operator which is the difference of U-shaped paths from quark to antiquark of the form $\sqcap-\sqcup$ ) was the lowest lying gluonic excitation. Results for this potential are shown in Fig. 3.

This gluonic excitation in the $E_{u}$ representation (or $\Pi_{u}$ in the continuum) corresponds to a component of angular momentum of one unit along the quark antiquark axis. Then one can solve for the spectrum of hybrid mesons using the Schrödinger equation in the adiabatic approximation. The spatial wave function necessarily has non zero angular momentum and the lightest states correspond to $L^{P C}=1^{+-}$and $1^{-+}$. Combining with the quark and antiquark spins then yields [17] a set of 8 degenerate hybrid states with $J^{P C}=1^{--}, 0^{-+}, 1^{-+}, 2^{-+}$and $1^{++}, 0^{+-}, 1^{+-}, 2^{+-}$respectively. These contain the spin-exotic states with $J^{P C}=1^{-+}, 0^{+-}$ and $2^{+-}$which will be of special interest.

Since the lattice calculation of the ground state and hybrid masses allows a direct prediction for their difference, the result for this 8-fold degenerate hybrid level is illustrated in Fig. 3 and corresponds [16] to masses of $10.81(25) \mathrm{GeV}$ for $b \bar{b}$ and $4.19(15) \mathrm{GeV}$ for $c \bar{c}$ when using the Schrödinger equation in the Born-Oppenheimer approximation to determine the spectrum. Here the errors take into account the uncertainty in setting the ground state mass using the quenched potential as discussed above. Recently a different lattice technique 18 has been used to explore in detail the excited gluonic levels in the quenched approximation. The results above are confirmed and the value quoted for the lightest hybrid meson 
is $\left(m_{\text {Hybrid }}-m_{\Upsilon}\right) r_{0}=3.166(3)$ for $b \bar{b}$ where the error does not take into account unquenching

The quenched lattice results, after adjusting to take account of the measured $\bar{b} b$ spectrum, suggest that the lightest hybrid mesons lie above the open $B \bar{B}$ threshold by about $270 \mathrm{MeV}$. This can also be studied by comparing directly the lattice hybrid masses with twice the quenched lattice masses for the $B$ meson [19]. Using quenched results from the smallest lattice spacing $(\beta=$ 6.2) available with clover-improved fermions [20] yields $m_{\text {Hybrid }}-2 m_{B} \approx 140(80) \mathrm{MeV}$. This estimate is somewhat smaller than that obtained above. In both cases, however, the hybrid levels lie above the open threshold and are likely to be relatively wide resonances. Another consequence [16] is that the very flat potential implies a very extended wavefunction. This is illustrated, for example, in ref [18] which shows that the hybrid wavefunction remains significant out to a radius of $1 \mathrm{fm}$. This has the implication that the wavefunction at the origin will be small, so hybrid vector states will be weakly produced from $e^{+} e^{-}$.

It would be useful to explore the splitting among the 8 degenerate $J^{P C}$ values obtained. This could come from different excitation energies in the $L^{P C}=1^{+-}$(magnetic) and $1^{-+}$(pseudoelectric) gluonic excitations, spin-orbit terms, as well as mixing between hybrid states and $Q \bar{Q}$ mesons with non-exotic spin. One way to study this on a lattice is to use the NRQCD formulation which describes non-static heavy quarks which propagate non-relativistically. Preliminary results for hybrid excitations from several groups [21, 22, 18] give at present similar results to those with the Born-Oppenheimer approximation in the static approximation as described above. There is some evidence that the magnetic excitations (which include the $1^{-+}$spin exotic) are lighter than the pseudo-electric ones (which include the $0^{+-}$spin exotic). For example ref[18] obtains $\left(m\left(1^{+-}\right)-m_{\Upsilon}\right) r_{0}=3.29(5)$ and $\left.\left(m^{-+}\right)-m_{\Upsilon}\right) r_{0}=3.51(8)$, to be compared with the static approximation, discussed above, which has degenerate levels with a value of $3.166(3)$. It has to be remembered that these are all quenched determinations, so there are system- atic errors of order $10 \%$ from unquenching. This implies that the NRQCD estimate for the lightest spin-exotic hybrid would be the $1^{+-}$state at a mass of 10.69(13) GeV.

\section{LIGHT QUARK INTERACTIONS}

Unlike very heavy quarks, light quark propagation in the gluonic vacuum sample is very computationally intensive - involving inversion of huge $\left(10^{9} \times 10^{9}\right)$ sparse matrices. Current computer power is sufficient to study light quark physics thoroughly in the quenched approximation. The state of the art 23] is the Japanese CP-PACS Collaboration who are able to study a range of large lattices (up to about $64^{4}$ ) with a range of light quark masses. Qualitatively the meson and baryon spectrum of states made of light and strange quarks is reproduced with discrepancies of order $10 \%$ in the quenched approximation.

Here I will focus on hybrid mesons made from light quarks. There will be no mixing with $q \bar{q}$ mesons for spin-exotic hybrid mesons and these are of special interest. The first study of this area was by the UKQCD Collaboration 10 who used operators motivated by the heavy quark studies referred to above. Using non-local operators, they studied all $8 J^{P C}$ values coming from $L^{P C}=1^{+-}$ and $1^{-+}$excitations. The resulting mass spectrum is shown in Fig. 4 where the $J^{P C}=1^{-+}$ state is seen to be the lightest spin-exotic state with a statistical significance of 1 standard deviation. The statistical error on the mass of this lightest spin-exotic meson is $7 \%$ but, to take account of systematic errors from the lattice determination, a mass of $2000(200) \mathrm{MeV}$ is quoted for this hybrid meson with $s \bar{s}$ light quarks. Although not directly measured, the corresponding light quark hybrid meson would be expected to be around $120 \mathrm{MeV}$ lighter. In view of the small statistical error, it seems unlikely that the $1^{-+}$ meson in the quenched approximation could lie as light as $1.4 \mathrm{GeV}$ where there are experimental indications for such a state [24]. Recently experimental evidence has also been presented [25] for another $1^{-+}$meson at $1.6 \mathrm{GeV}$.

One feature clearly seen in Fig. 4 is that non spin-exotic mesons created by hybrid meson oper- 


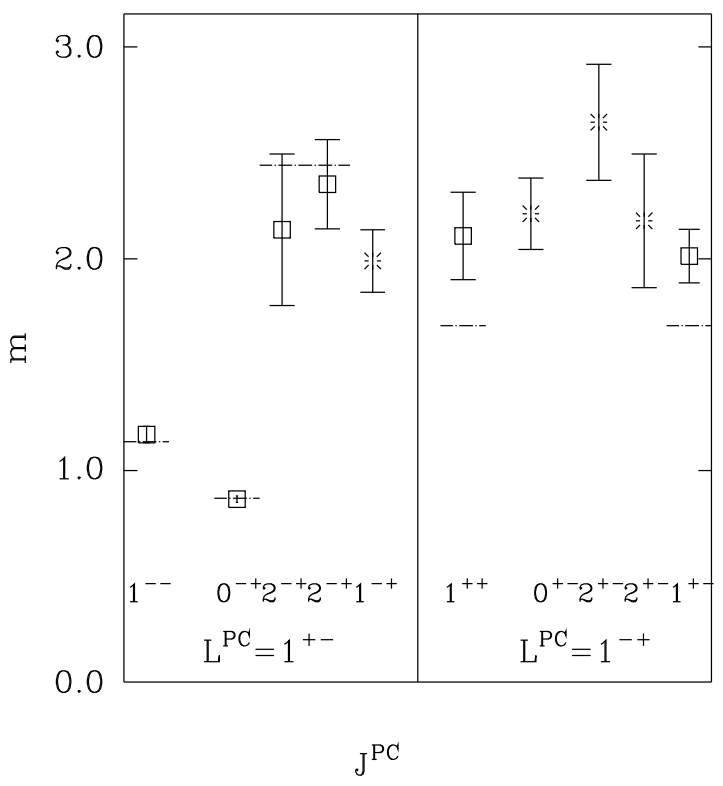

Figure 4. The masses in $\mathrm{GeV}$ of states of $J^{P C}$ built from hybrid operators with strange quarks, spin-exotic $(*)$ and non-exotic (squares). The dot-dashed lines are the mass values found for $s \bar{s}$ operators. Results from ref 10$]$.

ators have masses which are very similar to those found when the states are created by $q \bar{q}$ operators. This suggests that there is quite strong coupling between hybrid and $q \bar{q}$ mesons even in the quenched approximation. This would imply that the $\pi(1800)$ is unlikely to be a pure hybrid, for example.

A second lattice group has also evaluated hybrid meson spectra from light quarks from quenched lattices. They obtain [26] masses of the $1^{-+}$state with statistical and various systematic errors of 1970(90)(300) MeV, 2170(80)(100)(100) $\mathrm{MeV}$ and 4390(80)(200) MeV for $n \bar{n}, s \bar{s}$ and $c \bar{c}$ quarks respectively. For the $0^{+-}$spin-exotic state they have a noisier signal but evidence that it is heavier. They also explore mixing matrix elements between spin-exotic hybrid states and 4 quark operators.

Recently a first attempt has been made [27] to determine the hybrid meson spectrum using full QCD. The sea-quarks used have several different masses and an extrapolation is made to the limit of physical sea-quark masses, yielding a mass of $1.9(2) \mathrm{GeV}$ for the lightest spin-exotic hybrid meson, which they again find to be the $1^{-+}$. In principle this calculation should take account of sea-quark effects such as the mixing between such a hybrid meson and $q \bar{q} q \bar{q}$ states such as $\eta \pi$. As illustrated in Fig. 5. the calculations are performed for quite heavy sea quarks (the lightest being approximately the strange quark mass) and then a linear extrapolation is made. It is quite possible, however, that such mixing effects turn on nonlinearly as the sea-quark masses are reduced. The systematic error from this possibility is difficult to quantify.

The three independent lattice calculations of the light hybrid spectrum are in good agreement with each other. They imply that the natural energy range for spin-exotic hybrid mesons is around $1.9 \mathrm{GeV}$. The $J^{P C}=1^{-+}$state is found to be lightest. It is not easy to reconcile these lattice results with experimental indications for resonances at $1.4 \mathrm{GeV}$ and $1.6 \mathrm{GeV}$. Mixing with $q \bar{q} q \bar{q}$ states such as $\eta \pi$ is not included for realistic quark masses in the lattice calculations. This can be interpreted, dependent on one's viewpoint, as either that the lattice calculations are incomplete or as an indication that the experimental states may have an important meson-meson component in them.

\section{TOWARDS FULL QCD}

Algorithms exist which allow lattice simulation of full QCD with sea quarks of mass $m_{\text {sea }}$. This study needs lots of CPU power since the sea quark loops in the vacuum are represented effectively as a long range interaction between the gluonic degrees of freedom. Most studies to date have been exploratory with sea quark masses above the strange quark mass. In this regime, very little change from the quenched approximation is seen in physical predictions from the lattice.

One area where specific changes are expected is for the potential between heavy quarks. At small separation $R$, the Coulombic term is ex- 


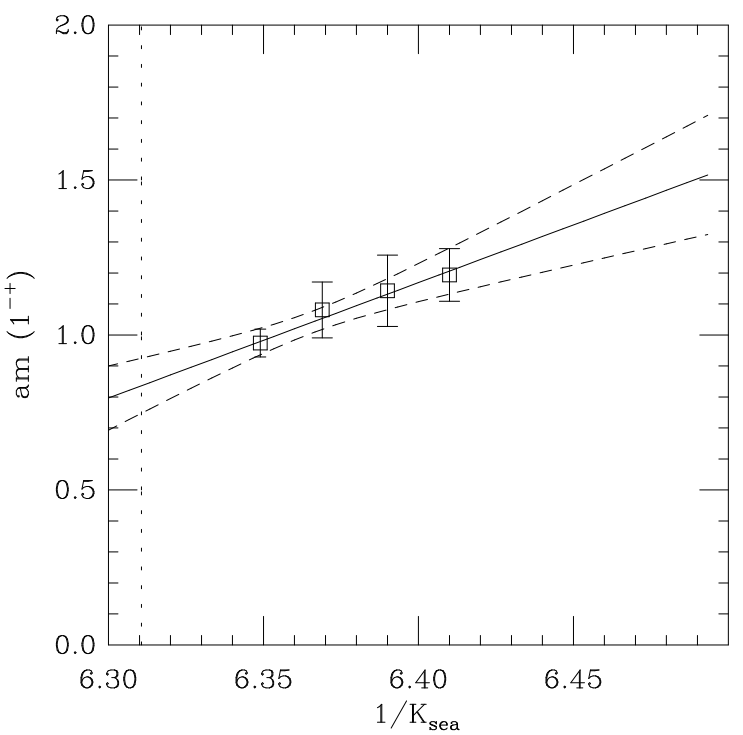

Figure 5. The extrapolation in sea-quark mass (in lattice units) from ref 27] for the $1^{-+}$hybrid meson. The dotted vertical line corresponds to light sea quarks.

pected to increase in strength and indeed evidence of this has been reported 28 30]. At larger $R$, signs of string breaking are expected since a light quark antiquark pair can be produced from the vacuum to yield two $Q \bar{q}$ mesons with energy independent of $R$ at large separation. This has been explored by the SESAM, UKQCD and CPPACS collaborations and little sign of the effect is seen [29,28,30].

It has been very difficult to see unambiguous signs of sea quark effects in the hadron spectrum. The CP-PACS collaboration 31 have recently reported that they see evidence that, for two flavours of light quark, results are closer to experiment than from quenched studies. Even their study, however, is restricted to sea-quark masses which are not appreciably lighter than the strange quark mass. Now, it is possible that such sea-quark effects turn on non-linearly as the sea quark mass is reduced. As an example, in cur- rent studies the $\pi+\pi \mathrm{P}$-wave is heavier than the $\rho$ meson so the $\rho$ cannot decay. Another example is that glueball mixing with $q \bar{q}$ scalar mesons may depend quite sensitively on their masses, so as the sea quark mass is reduced, the scalar meson mass will move relative to the glueball mass so causing a non-linear change in the mixing strength.

Further work is needed to reduce the sea quark mass and to increase the lattice size. Dedicated computing power of several hundreds of Gflops is available to lattice collaborations and, with future plans for Tflops machines, progress in this area should now be possible.

\section{SUMMARY}

Quenched lattice QCD is well understood and accurate predictions in the continuum limit are increasingly becoming available. Glueball masses of $m\left(0^{++}\right)=1611(30)(160) \mathrm{MeV}$; $m\left(2^{++}\right)=2232(220)(220) \mathrm{MeV}$ and $m\left(0^{-+}\right)=$ $2611(370)(260) \mathrm{MeV}$ are predicted where the second error is an overall scale error. The quenched approximation also gives information on quarkantiquark scalar mesons and their mixing with glueballs. This determination of the mixing in the quenched approximation is currently more powerful than attempting to determine the mixed spectrum directly in full QCD because the sea-quark masses realisable are rather heavy. There is also some lattice information on the hadronic decay amplitudes of glueballs.

For hybrid mesons, there will be no mixing with $q \bar{q}$ for spin-exotic states and these are the most useful predictions. The $J^{P C}=1^{-+}$state is expected at $10.81(25) \mathrm{GeV}$ for $b$ quarks; $4.19(15)$ $\mathrm{GeV}$ for $c$ quarks, 2.0(2) GeV for $s$ quarks and $1.9(2) \mathrm{GeV}$ for $u, d$ quarks. Mixing of spin-exotic hybrids with $q \bar{q} q \bar{q}$ or equivalently with mesonmeson is allowed and will modify the predictions from the quenched approximation.

Much activity is currently underway to explore the effects of sea quarks of ever decreasing mass. Future teraflops computing facilities will be essential to obtain quantitative results for hadronic spectroscopy. 


\section{REFERENCES}

1. K.G. Wilson, Phys. Rev. D10 (1974) 2445.

2. C. Michael, Nucl. Phys. B327 (1989) 517.

3. SESAM Collaboration, G. Bali et al., Nucl. Phys. B (Proc. Suppl.) 53 (1997) 239.

4. P. De Forcrand et al., Phys. Lett. 152B (1985) 107.

5. C. Michael and M. Teper, Nucl. Phys. B314 (1989) 347.

6. UKQCD collaboration, G. Bali, et al., Phys. Lett. B309 (1993) 378.

7. H. Chen et al., Nucl. Phys. B (Proc. Suppl.) 34 (1994) 357.

8. C. Morningstar and M. Peardon, Phys. Rev. D56 (1997) 4043.

9. C. Morningstar and M. Peardon, contribution to LAT98, hep-lat/9808045.

10. UKQCD Collaboration, P. Lacock, C. Michael, P. Boyle and P. Rowland, Phys. Rev. D54 (1996) 6997; Phys. Lett. B401 (1997) 308; Nucl. Phys. B (Proc. Suppl.) 63 (1998) 203.

11. W. Lee and D. Weingarten, Nucl. Phys. B (Proc. Suppl) 53 (1997) 236;

12. W. Lee and D. Weingarten, Nucl. Phys. B (Proc. Suppl) 63 (1998) 194; hep-lat/9805029.

13. D. Weingarten, Nucl. Phys. B (Proc. Suppl) 53 (1997) 232

14. F. Close, Nucl. Phys. A623 (1997) 125c.

15. J. Sexton, A. Vaccarino and D. Weingarten, Nucl. Phys. B (Proc. Suppl.) 42 (1995) 279.

16. S. Perantonis and C. Michael, Nucl. Phys. B347 (1990) 854.

17. L.A. Griffiths, C. Michael and P.E.L. Rakow, Phys. Lett. 129B (1983) 351.

18. K. Juge, J. Kuti and C. Morningstar, Nucl. Phys. B (Proc. Suppl.) 63 (1998) 326; Hadron Spectroscopy, Seventh International Conference, AIP New York 1998, ed. Chung and Willutzki, 137, hep-ph/9711451; contribution to Confinement III, hep-lat/9809015; contribution to LAT98, hep-lat/9809098.

19. R. Sommer, Phys. Rep. 275 (1996) 1.

20. UKQCD Collaboration, A. Ewing et al., Phys. Rev. D54 (1996) 3526.

21. UKQCD Collaboration, T. Manke et al., Nucl. Phys. B (Proc. Suppl.) 63 (1998) 332; hep-lat/9710083.

22. UKQCD Collaboration, S. Collins et al., Nucl. Phys. B (Proc. Suppl.) 63 (1998) 335.

23. T. Yoshie, Nucl. Phys. B (Proc. Suppl.) 63 (1998) 3; CP-PACS Collaboration, S. Aoki et al., contribution to LAT98, hep-lat/9809146.

24. D. Thompson et al., Phys. Rev. Lett. 79 (1997) 1630; A. Ostrovidov, Hadron Spectroscopy, Seventh International Conference, AIP New York 1998, ed. Chung and Willutzki, 263.

25. D. Weygand, contribution to Confinement III, TJ Lab, 1998.

26. C. Bernard et al., Nucl. Phys. B (Proc. Suppl.) 53 (1996) 228; Phys. Rev. D56 (1997) 7039; hep-lat/9809087.

27. SESAM Collaboration, P. Lacock and K. Schilling, hep-lat/9809022.

28. SESAM Collaboration, U. Glässner et al., Phys. Lett. B383 (1966) 98; S. Güsken, Nucl. Phys. B (Proc. Suppl.) 63 (1998) 16.

29. UKQCD Collaboration, C. Allton et al., heplat/9808016.

30. CP-PACS Collaboration, S. Aoki et al., heplat/9809185.

31. CP-PACS Collaboration, S. Aoki et al., heplat/9809120. 